\title{
Adaptação à universidade e transtornos mentais comuns em graduandos de enfermagem
}

\author{
Cíntia Tavares Carleto ${ }^{1}$ \\ Raysa Cristina Dias de Moura² \\ Virgínia Souza Santos ${ }^{3}$ \\ Leila Aparecida Kauchakje Pedrosa ${ }^{4}$
}

\section{RESUMO}

Objetivou-se avaliar a adaptação à universidade e a sua relação com a ocorrência de Transtornos Mentais Comuns (TMC) em graduandos de enfermagem. Estudo analítico, transversal, com abordagem quantitativa dos dados, realizado com 92 graduandos de enfermagem de uma Instituição Federal de Ensino Superior de Minas Gerais. Para coleta de dados foram utilizados um questionário sociodemográfico e acadêmico, o Questionário de Vivências Acadêmicas-reduzido (QVA-r) e o Self-Reporting Questionnaire (SRQ-20). Observou-se bom nível de adaptação entre os participantes, com melhores escores na dimensão Carreira e escores inferiores na dimensão Pessoal. Encontrou-se um indicativo de TMC de 43,5\% e uma correlação inversa com adaptação à universidade, indicando que quanto melhor a adaptação, menor a probabilidade de TMC. Os achados evidenciaram que o processo de adaptação à universidade está relacionado à saúde mental dos graduandos e indicam a necessidade de intervenções direcionadas à adaptação acadêmica como estratégia de promoção da saúde.

Descritores: Educação Superior; Estudantes de Enfermagem; Adaptação; Transtornos Mentais; Saúde Mental.

\footnotetext{
${ }^{1}$ Enfermeira, Doutora em Atenção à Saúde. Enfermeira do Núcleo de Assistência Estudantil em Saúde da Universidade Federal do Triângulo Mineiro. Uberaba, MG, Brasil. E-mail: carletoctc@gmail.com.

2 Enfermeira. Uberaba, MG, Brasil. E-mail: raysacristina_moura@hotmail.com.

${ }^{3}$ Nutricionista, Doutora em Atenção à Saúde. Professora Assistente da Universidade Federal de Viçosa, Campus de Rio Paranaíba. Rio Paranaíba, MG, Brasil. E-mail: virginiasantos@ufv.br.

${ }^{4}$ Enfermeira, Doutora em Enfermagem, Professora Associada da Universidade Federal do Triângulo Mineiro. Uberaba, MG, Brasil. E-mail: leila.kauchakje@enfermagem.uftm.edu.br.
}

Artigo recebido: 28/10/2016.

Artigo aprovado: 08/12/2017.

Artigo publicado: 17/04/2018.

\section{Como citar esse artigo:}

Carleto CT, Moura RCD, Santos VS, Pedrosa LAK. Adaptação à universidade e transtornos mentais comuns em graduandos de enfermagem. Rev. Eletr. Enf. [Internet]. 2018 [acesso em: ];20:v20a01. Disponível em: http://doi.org/10.5216/ree.v20.43888. 


\section{INTRODUÇÃO}

O ingresso na universidade configura-se como um momento de satisfação para o estudante, devido à possibilidade de concretização de sua formação profissional. No entanto, apesar de motivado pela busca do diploma, não significa que o estudante esteja completamente preparado para enfrentar esse desafio. Muitas vezes, o estudante pode encontrar dificuldades para se adaptar a esse novo ambiente em virtude das demandas relacionadas ao curso e à instituição, às relações interpessoais e às questões emocionais, como o estresse e ansiedade $^{(1-2)}$.

A vida universitária corresponde a um período de grandes mudanças na vida do estudante, sendo que estas são responsáveis por inúmeras situações estressantes, como a distância da família, novos relacionamentos interpessoais, relacionamentos amorosos, a adaptação à vida acadêmica, decisão sobre prioridades e gerenciamento da vida financeira ${ }^{(3)}$. As situações estressantes aumentam progressivamente, uma vez que a cada etapa do curso surgem novas exigências que requerem o desenvolvimento de habilidades e competências por parte dos estudantes ${ }^{(4)}$.

Juntamente com a obtenção de novos conhecimentos, a vida universitária proporciona o contato com novos valores e crenças, questionamentos e experiências acadêmicas e sociais que propiciam aos estudantes um amadurecimento pessoal e que irão repercutir sobre a profissão escolhida e sobre a constituição individual dos estudantes ${ }^{(5)}$. Além disso, o meio universitário pode representar para o discente tanto um ambiente promotor de saúde quanto um espaço limitante desta, com possibilidades de estresse negativo, por ser um local onde o estudante permanece grande parte do dia, por vários anos, convive com uma diversidade de pessoas e com situações que o levam a desenvolver estratégias de sobrevivência da forma mais saudável possível(6).

Dessa forma, a adaptação à vida universitária não é um processo fácil e as repercussões deste processo que, muitas vezes, pode levar ao insucesso acadêmico, vão além da área da educação e incidem, diretamente, sobre a saúde do indivíduo e da comunidade e no desenvolvimento social e econômico do país(7).

Os estudantes da área da saúde experimentam altos níveis de estresse, cujas manifestações, psicológicas e fisiológicas, acontecem em maior proporção entre graduandos de enfermagem ${ }^{(8)}$. Estes, muitas vezes, sentem-se vulneráveis às diversas demandas universitárias, como os problemas relativos à qualidade do ensino e ao ambiente educacional, situações pessoais, o planejamento do futuro profissional, a organização frente ao crescente volume de informações, o estresse resultante das atividades práticas, além do desgaste decorrente do contato com pessoas doentes e com a morte ${ }^{(9)}$.

Estas e outras situações, como as dificuldades adaptativas, podem predispor ao aparecimento dos Transtornos Mentais Comuns (TMC) que se referem a quadros menos graves de transtorno mental e incluem sintomas como esquecimento, insônia, irritabilidade, dificuldade na concentração e tomada de decisões, fadiga e queixas somáticas, como cefaleia, falta de apetite, tremores, entre outras ${ }^{(10)}$. Os TMC podem ser evidenciados desde o ingresso do estudante no ensino superior e são mais frequentes entre os universitários da área da Saúde, uma vez que, em sua rotina acadêmica, lidam com o sofrimento e a dor ${ }^{(11)}$.

Na maioria das vezes, os TMC não são identificados ou tratados corretamente e tendem a ser subestimados pelos profissionais de saúde, especialmente na ausência de sintomas físicos, e, assim, podem evoluir e se tornarem 
crônicos. O diagnóstico precoce e correto desses transtornos pode prevenir os prejuízos físicos e psicológicos aos indivíduos e o ônus ao sistema de saúde ${ }^{(12)}$.

No contexto universitário, os problemas de saúde mental entre os estudantes têm aumentado em número e gravidade e constituem grande desafio para as instituições de ensino superior e para os serviços de saúde. Sintomas como estresse, ansiedade, entre outros, relacionados às vivências acadêmicas, podem impactar negativamente a saúde mental, o desempenho acadêmico, o desenvolvimento do estudante e acarretar consequências para sua formação e atuação futuras ${ }^{(9,13)}$.

Nesse sentido, compreende-se que o estudante de enfermagem que futuramente prestará cuidado, necessita de atenção e cuidado, a fim de manter sua saúde física e mental em níveis adequados e satisfatórios, de modo que venha a refletir em sua futura atuação profissional ${ }^{(4,14-15)}$.

Diante desse contexto e considerando a escassez de estudos sobre a relação entre as temáticas, este estudo teve como objetivo avaliar a adaptação à universidade e a sua relação com a ocorrência de TMC em graduandos de enfermagem.

\section{MÉTODOS}

Estudo exploratório, analítico, transversal, com abordagem quantitativa dos dados, realizado com graduandos de Enfermagem de uma Instituição Federal de Ensino Superior de Minas Gerais, no período de novembro de 2015 a fevereiro de 2016.

O cálculo do tamanho amostral considerou um coeficiente de determinação apriorístico de $R^{2}=0,13$, em um modelo de regressão linear com três preditores, tendo como nível de significância ou erro do tipo I de $\alpha=0,05$ e erro do tipo II de $\beta=0,1$, resultando, portanto, em um poder estatístico apriorístico de 90,0\%. Utilizou-se o aplicativo PASS (Power Analysis and Sample Size), versão de 2002, e obteve-se um tamanho de amostra de n=99 sujeitos que foram sorteados, aleatoriamente, entre os 275 estudantes regularmente matriculados no curso de graduação em Enfermagem da instituição.

Como critério de inclusão considerou-se a idade igual ou superior a 18 anos e foram excluídos os estudantes em licença, afastamento ou trancamento do curso no período da coleta de dados. Ao final, obteve-se uma participação de 92 graduandos, ocasionando uma perda amostral de sete sujeitos (7,1\%).

Para a coleta de dados foram utilizados três instrumentos autoaplicáveis: um questionário sociodemográfico e acadêmico, o Questionário de Vivências Acadêmicas-reduzido (QVA-r) e o Self-Reporting Questionnaire (SRQ-20).

O questionário sociodemográfico e acadêmico foi elaborado pelos pesquisadores e possuía variáveis de caracterização dos sujeitos: data de nascimento, sexo, estado civil, cor da pele, número de filhos, doenças crônicas, vínculo empregatício e carga horária de trabalho semanal, rendas pessoal e familiar mensal, ano de ingresso no curso de graduação, período que está cursando e retenção em disciplinas.

O Questionário de Vivências Acadêmicas é um instrumento de autorrelato, construído em Portugal, que tem por objetivo avaliar a percepção dos estudantes a respeito de suas experiências acadêmicas na universidade e inferir sobre a qualidade da sua adaptação à universidade. Apresenta-se em duas versões, uma versão integral (QVA) e uma versão reduzida (QVA-r) $)^{(16)}$. 
O QVA-r foi validado para uso no Brasil em 2005 e contém 55 itens distribuídos em cinco dimensões: Pessoal (bem estar físico e psicológico, equilíbrio emocional, estabilidade afetiva, otimismo e autoconfiança); Interpessoal (relações com colegas, competências de relacionamento em situações de maior intimidade, estabelecimento de amizades e procura de ajuda); Carreira (sentimentos relacionados com o curso, perspectivas de carreira e projetos vocacionais); Estudo (hábitos de estudo, gestão do tempo, utilização dos recursos de aprendizagem no campus e preparação para os testes) e Institucional (apreciação dos alunos face à instituição de ensino que frequentam, desejo de permanecer ou mudar de instituição, conhecimento e apreciação das infraestruturas existentes) ${ }^{(16)}$. Os itens devem ser avaliados considerando a trajetória acadêmica até o momento atual e são respondidos em uma escala do tipo Likert de cinco pontos. Nesta pesquisa, os escores de cada subescala foram calculados considerando os níveis de adaptação variando de um a cinco, sendo o menor valor relacionado ao nível mais baixo e o maior valor ao mais alto nível de adaptação.

O SRQ-20 foi desenvolvido pela Organização Mundial de Saúde para mensuração do nível de suspeição de transtornos mentais e possui caráter de triagem, ou seja, destina-se à detecção de sintomas e não oferece diagnóstico do tipo de transtorno existente. Os sintomas avaliados pelo SRQ-20 aproximam-se dos TMC e são caracterizados como não psicóticos: fadiga, irritabilidade, insônia, dificuldade de concentração, esquecimento e queixas somáticas $^{(17)}$.

Os itens devem ser avaliados considerando dores e problemas nos últimos 30 dias. As respostas do questionário são dicotômicas e cada resposta positiva recebe o valor de um ponto, cuja soma compõe o escore final. Os escores obtidos são referentes à probabilidade de presença de transtorno não-psicótico e varia de zero a 20, sendo nenhuma e extrema probabilidade de apresentar TMC, respectivamente. Como ponto de corte foi utilizado o escore total igual ou menor a sete como caso negativo e igual ou maior a oito como rastreamento positivo para $\operatorname{TMC}^{(17)}$.

Os dados foram analisados estatisticamente por meio do programa Statistical Package for the Social Science (SPSS), versão 21.0. Foram realizadas análises descritivas dos dados a partir da apuração de frequências simples absolutas e percentuais para as variáveis categóricas e medidas de centralidade (média, mediana, moda) e de dispersão (desvio padrão, mínimo e máximo) para variáveis quantitativas. Nas análises bivariadas utilizou-se um nível de significância de 5,0\% (p $\leq 0,05)$ e foi realizado o teste t de Student a fim de comparar variáveis quantitativas entre grupos definidos por variáveis categóricas e verificar se os resultados obtidos foram estatisticamente significativos. Realizou-se a correlação de Pearson entre os escores do QVA-r e do SRQ-20 e foram classificadas como fracas $(0<r<0,3)$, moderadas $(0,3 \leq r<0,5)$ ou fortes $(r \geq 0,5)$.

Ressalta-se que este estudo integra uma pesquisa maior intitulada "Promoção e proteção da saúde física e mental de estudantes universitários" que foi aprovada pelo Comitê de Ética em Pesquisa com Seres Humanos da instituição, conforme parecer no 1.226 .066 e CAAE 44557015.3.0000.5154, e conduzida de acordo com as diretrizes e normas brasileiras regulamentadoras de pesquisas envolvendo seres humanos. A participação dos estudantes ocorreu após anuência e assinatura do Termo de Consentimento Livre e Esclarecido. 


\section{RESULTADOS}

Participaram deste estudo 92 graduandos de enfermagem, sendo a maioria representada pelo sexo feminino $(88,0 \%, n=81)$, de cor branca $(64,1 \%, n=59)$, com idade variando de 18 a 43 anos completos, sendo a média de 22,26 anos (mediana 22,0 anos, desvio-padrão 3,34 anos), estado civil solteiro (94,6\%, $\mathrm{n}=87$ ), procedente da cidade onde o estudo foi realizado $(62,0 \%, n=57)$, sem vínculo empregatício $(92,4 \%, n=85)$.

Os períodos do curso foram recodificados em dois grupos: $25,0 \%(n=23)$ eram alunos ingressantes (primeiro e segundo períodos) e $75,0 \%(n=69)$ cursavam os demais períodos.

Ao analisar os resultados das médias do QVA-r, observou-se que as melhores médias foram obtidas nas dimensões Carreira $(3,85)$, Institucional $(3,79)$ e Interpessoal $(3,77)$. As dimensões Estudo e Pessoal apresentaram médias inferiores, 3,52 e 3,32 respectivamente, conforme Tabela 1.

A consistência interna do Questionário de Vivências Acadêmicas (QVA-r) foi avaliada utilizando-se o coeficiente alfa de Cronbach, possibilitando avaliar as respostas dos participantes com um bom grau de fidedignidade (Tabela 1).

Tabela 1: Medidas de centralidade, dispersão e consistência interna das dimensões do QVA-r entre graduandos de Enfermagem de uma Instituição Federal de Ensino Superior de Minas Gerais, Brasil, 2016.

\begin{tabular}{cccccc}
\hline Dimensões QVA-r & Média & Desvio padrão & Mínimo & Máximo & alfa de Cronbach \\
\hline Pessoal & 3,32 & 0,76 & 1,29 & 5,00 & 0,88 \\
Interpessoal & 3,77 & 0,73 & 1,33 & 5,00 & 0,88 \\
Carreira & 3,85 & 0,69 & 2,17 & 5,00 & 0,86 \\
Estudo & 3,52 & 0,79 & 1,44 & 5,00 & 0,87 \\
Institucional & 3,79 & 0,67 & 1,88 & 4,88 & 0,76 \\
\hline
\end{tabular}

Os estudantes procedentes do local do estudo apresentaram melhores escores de Vivências Acadêmicas na dimensão Institucional que os alunos procedentes de outras cidades, cujas diferenças foram significativas $(p=0,043)$. As demais variáveis sociodemográficas e acadêmicas não apresentaram diferenças estatisticamente significativas.

Entre os estudantes participantes deste estudo detectou-se uma prevalência de 43,5\% de rastreamento positivo para TMC. Os sintomas avaliados foram agrupados em quatro categorias, conforme Tabela 2.

Na categoria "Humor depressivo/ansioso", o sintoma mais relatado pelos estudantes foi sentir-se nervoso, tenso ou preocupado (83,7\%). Na categoria "Sintomas somáticos", dormir mal (53,3\%) e cansar-se com facilidade $(50,0 \%)$ foram os mais relatados. No grupo “Decréscimo de energia vital”, a dificuldade de tomar decisões foi a mais relatada (44,6\%), seguida de sentir-se cansado o tempo todo $(40,2 \%)$ e no grupo "Pensamentos depressivos", perder o interesse pelas coisas representou $35,9 \%$ das respostas.

Observou-se maior ocorrência de TMC entre os estudantes do sexo masculino (45,8\%) em relação às mulheres (43,2\%), no entanto, não foram observadas diferenças significativas.

Os estudantes procedentes do local do estudo apresentaram maior ocorrência de TMC (50,9\%) em relação aos migrantes $(31,4 \%)$, no entanto, as diferenças não foram significativas ( $p=0,06, R P=1,615, I C=0,932-2,811)$. 
Tabela 2: Prevalência dos sintomas de TMC em graduandos de Enfermagem de uma Instituição Federal de Ensino Superior de Minas Gerais, de acordo com o grupo de sintomas do SRQ-20, Brasil, 2016.

\begin{tabular}{|c|c|c|}
\hline \multirow{2}{*}{ Grupo de sintomas - SRQ-20 } & Sim & Não \\
\hline & $\%(n)$ & $\%(n)$ \\
\hline \multicolumn{3}{|l|}{ Humor depressivo/ansioso } \\
\hline Q-4 Assusta-se com facilidade? & $45,7(42)$ & $54,3(50)$ \\
\hline Q-6 Sente-se nervoso (a), tenso (a) ou preocupado (a)? & $83,7(77)$ & $16,3(15)$ \\
\hline Q-9 Tem se sentido triste ultimamente? & $41,3(38)$ & $58,7(54)$ \\
\hline Q-10 Tem chorado mais do que costume? & $30,4(28)$ & $69,6(64)$ \\
\hline \multicolumn{3}{|l|}{ Sintomas somáticos } \\
\hline Q-1 Você tem dores de cabeça frequente? & $49,0(45)$ & $51,0(47)$ \\
\hline Q-2 Tem falta de apetite? & $14,0(13)$ & $86,0(79)$ \\
\hline Q-3 Dorme mal? & $53,3(49)$ & $46,7(43)$ \\
\hline Q-5 Tem tremores nas mãos? & $18,5(17)$ & $81,5(75)$ \\
\hline Q-7 Tem má digestão? & $21,7(20)$ & $78,3(72)$ \\
\hline Q-19 Você se cansa com facilidade? & $50,0(46)$ & $50,0(46)$ \\
\hline \multicolumn{3}{|l|}{ Decréscimo de energia vital } \\
\hline Q-8 Tem dificuldades de pensar com clareza? & $34,8(32)$ & $65,2(60)$ \\
\hline $\begin{array}{l}\text { Q-11 Encontra dificuldades para realizar com } \\
\text { satisfação suas atividades diárias? }\end{array}$ & $33,7(31)$ & $66,3(61)$ \\
\hline Q-12 Tem dificuldades para tomar decisões? & $44,6(41)$ & $55,4(51)$ \\
\hline $\begin{array}{l}\text { Q-13 Tem dificuldades no serviço (seu trabalho é penoso, causa-lhe } \\
\text { sofrimento?) }\end{array}$ & $12,0(11)$ & $88,0(81)$ \\
\hline Q-18 Sente-se cansado (a) o tempo todo? & $40,2(37)$ & $59,8(55)$ \\
\hline Q-20 Tem sensações desagradáveis no estômago? & $35,9(33)$ & $64,1(59)$ \\
\hline \multicolumn{3}{|l|}{ Pensamentos depressivos } \\
\hline Q-14 É incapaz de desempenhar um papel útil em sua vida? & $20,7(19)$ & $79,3(73)$ \\
\hline Q-15 Tem perdido o interesse pelas coisas? & $35,9(33)$ & $64,1(59)$ \\
\hline Q-16 Você se sente uma pessoa inútil, sem préstimo?* & $15,2(14)$ & $83,7(77)$ \\
\hline Q-17 Tem tido ideia de acabar com a vida? & $4,3(04)$ & $95,7(88)$ \\
\hline
\end{tabular}

* Não respondeu: $01(1,1 \%)$

Os alunos ingressantes (primeiro e segundo períodos) apresentaram maior ocorrência de TMC (60,9\%) em relação aos dos demais períodos $(37,7 \%)$ e as diferenças foram marginalmente significativas $(p=0,052, R P=1,615$, IC $=1,034-2,525)$.

A Tabela 3 apresenta a comparação dos escores dicotomizados de TMC e de Vivências Acadêmicas. Observou-se escores inferiores de Vivências Acadêmicas entre os estudantes com rastreamento positivo para TMC, quando comparados aos estudantes com rastreamento negativo. Da mesma forma, os estudantes com melhores escores de Vivências Acadêmicas não apresentaram TMC.

De fato, evidenciou-se uma correlação inversa, estatisticamente significativa, entre os escores de Vivências Acadêmicas e de TMC (Tabela 4), indicando que quanto melhor a adaptação à universidade, menor a predisposição dos estudantes aos TMC. Observaram-se correlações fracas entre TMC e as dimensões Interpessoal $(-0,22)$ e Institucional $(-0,22)$, moderadas entre TMC e as dimensões Carreira $(-0,31)$ e Estudo $(-0,39)$ e forte entre TMC e a dimensão Pessoal $(-0,77)$. 
Tabela 3: Comparação entre os escores de Vivências Acadêmicas e de TMC de graduandos de enfermagem de uma Instituição Federal de Ensino Superior de Minas Gerais, Brasil, 2016.

\begin{tabular}{|c|c|c|}
\hline \multirow{2}{*}{ Vivências Acadêmicas } & \multicolumn{2}{|c|}{ TMC } \\
\hline & Sim & Não \\
\hline \multicolumn{3}{|l|}{ Dimensão Pessoal } \\
\hline Média & 2,71 & 3,79 \\
\hline Desvio padrão & 0,50 & 0,55 \\
\hline Mínimo & 1,29 & 2,79 \\
\hline Máximo & 3,64 & 5,00 \\
\hline \multicolumn{3}{|l|}{ Dimensão Interpessoal } \\
\hline Média & 3,65 & 3,85 \\
\hline Desvio padrão & 0,78 & 0,69 \\
\hline Mínimo & 1,33 & 1,50 \\
\hline Máximo & 4,92 & 5,00 \\
\hline \multicolumn{3}{|l|}{ Dimensão Carreira } \\
\hline Média & 3,64 & 4,01 \\
\hline Desvio padrão & 0,73 & 0,62 \\
\hline Mínimo & 2,25 & 2,17 \\
\hline Máximo & 4,92 & 5,00 \\
\hline \multicolumn{3}{|l|}{ Dimensão Estudo } \\
\hline Média & 3,27 & 3,73 \\
\hline Desvio padrão & 0,87 & 0,65 \\
\hline Mínimo & 1,44 & 2,11 \\
\hline Máximo & 5,00 & 5,00 \\
\hline \multicolumn{3}{|l|}{ Dimensão Institucional } \\
\hline Média & 3,66 & 3,88 \\
\hline Desvio padrão & 0,70 & 0,63 \\
\hline Mínimo & 2,25 & 4,88 \\
\hline Máximo & 1,88 & 4,88 \\
\hline
\end{tabular}

Tabela 4: Correlação entre os escores de Vivências Acadêmicas e de TMC de graduandos de enfermagem de uma Instituição Federal de Ensino Superior de Minas Gerais, Brasil, 2016.

\begin{tabular}{ccc}
\hline \multirow{2}{*}{ Vivências Acadêmicas (QVA-r) } & \multicolumn{2}{c}{ TMC (SRQ-20) } \\
\cline { 2 - 3 } & $\mathbf{r}$ & $\mathbf{p}$ \\
\hline Dimensão Pessoal & $-0,77$ & $<0,001$ \\
Dimensão Interpessoal & $-0,22$ & 0,032 \\
Dimensão Carreira & $-0,31$ & 0,003 \\
Dimensão Estudo & $-0,39$ & $<0,001$ \\
Dimensão Institucional & $-0,22$ & 0,033 \\
\hline
\end{tabular}

* Coeficiente de correlação de Pearson

\section{DISCUSSÃO}

O perfil dos graduandos deste estudo assemelha-se ao encontrado em outras pesquisas brasileiras que descrevem maior número de estudantes de Enfermagem como adultos jovens, do sexo feminino e estado civil solteiro, tanto em instituições públicas como privadas ${ }^{(18-19)}$.

Em relação à adaptação ao contexto universitário, verificou-se que os participantes deste estudo pontuaram acima do escore médio da escala ${ }^{(3)} \mathrm{em}$ todas as dimensões, demonstrando bom nível de adaptação. 
As melhores médias foram obtidas nas dimensões Carreira, Institucional e Interpessoal, enquanto médias inferiores foram obtidas nas dimensões Estudo e Pessoal.

Estudos realizados com universitários de outros cursos também apontam a dimensão Carreira como a que obteve a melhor média entre os alunos e a dimensão Pessoal como uma das médias mais baixas ${ }^{(1)}$.

A dimensão Carreira possui grande importância para o desenvolvimento da identidade e da adaptação do estudante à vida universitária, pois está relacionada ao curso escolhido, aos projetos vocacionais e à realização profissional. Já a dimensão Pessoal agrupa variáveis relacionadas ao bem-estar físico e psicológico do estudante, abrangendo hábitos de sono, saúde, autonomia, autoconfiança, otimismo, satisfação com a vida, concentração, equilíbrio emocional, dentre outros(1).

Assim, podemos inferir que, possivelmente, os estudantes desta pesquisa estão mais envolvidos com as aprendizagens no curso e as perspectivas de carreira, estão interessados pela instituição e desejam nela prosseguir, conhecem os serviços e estruturas existentes, relacionam-se com seus pares e envolvem-se em atividades extracurriculares. Porém, precisam melhorar suas competências de estudo, a gestão do tempo, a utilização dos diversos recursos de aprendizagem e, especialmente, suas percepções de bem-estar físico e psicológico ${ }^{(16)}$.

Os participantes procedentes do local do estudo apresentaram melhor adaptação ao contexto universitário na dimensão Institucional quando comparados aos estudantes de outras cidades. Este resultado pode estar relacionado ao fato de os estudantes possuírem maior conhecimento sobre a universidade, à qualidade dos serviços e estruturas existentes e desejarem nela prosseguir com seus estudos ${ }^{(16)}$.

Em relação aos TMC, a prevalência encontrada neste estudo $(43,5 \%)$ pode ser considerada alta quando comparada a outros estudos brasileiros com estudantes de enfermagem ou de outros $\operatorname{cursos}^{(11,20-22)}$.

Estudo realizado com estudantes de Biologia, Enfermagem, Fisioterapia e Psicologia, no Rio Grande do Sul, identificou que 20,0\% dos participantes apresentaram predisposição para TMC, sendo que a maior ocorrência foi observada entre os alunos do curso de Fisioterapia $(40,0 \%)$ e de Enfermagem $(25,0 \%)^{(11)}$.

Em Alagoas, pesquisa com universitários da área da saúde também encontrou elevada classificação positiva para TMC entre os participantes, na ordem de 43,2\%. No entanto, na estratificação por cursos, os graduandos de enfermagem apresentaram uma prevalência de 22,1\% de $\operatorname{TMC}^{(20)}$.

Outro estudo realizado com universitários de Psicologia e Enfermagem identificou uma ocorrência de fatores indicativos de TMC em 35,71\% dos estudantes ${ }^{(21)}$.

Recente estudo com 134 estudantes de Medicina, do primeiro ao quarto ano, de uma universidade pública do Sul do Brasil, identificou altas prevalências de TMC, sendo de 35,8\% no início do semestre e de 51,5\% no final do semestre ${ }^{(22)}$.

A alta prevalência de rastreio positivo para TMC encontrada neste e nos demais estudos brasileiros aponta uma preocupante situação de saúde mental entre os estudantes, especialmente os da área da Saúde, e indica a necessidade da identificação precoce e intervenção adequada frente aos TMC. Além disso, devem ser implementadas medidas de prevenção direcionadas aos universitários, como serviços de apoio psicopedagógico, ações que favoreçam um ambiente mais saudável e melhores relações interpessoais, orientação e conscientização para o cuidado com a saúde física e mental(14). 
Quanto ao período do curso, embora as diferenças encontradas tenham sido marginalmente significativas, observou-se maior ocorrência de TMC entre os estudantes ingressantes quando comparados aos dos demais períodos. Resultados semelhantes foram encontrados entre universitários da área da Saúde, em que, descritivamente, houve maior predisposição aos TMC na fase de admissão acadêmica (43,0\%) em relação às etapas intermediária $(28,0 \%)$ e final do curso $(28,0 \%)^{(11)}$.

Estudo internacional realizado com 308 universitários verificou associação entre a variável nível acadêmico e estresse $(r=0,12, p<0,05)$, indicando que os estudantes ingressantes ou do segundo ano estavam mais propensos a sintomas de estresse do que os dos demais níveis dos $\operatorname{cursos}^{(23)}$. Estes dados levam à reflexão de que os estudantes ingressantes podem estar mais susceptíveis aos TMC, em virtude das situações estressantes relacionadas à entrada na vida universitária, à necessidade de se adaptarem ao novo contexto e às diversas demandas deste.

Nesta pesquisa, embora não tenham sido encontradas diferenças significativas, observou-se, descritivamente, maior ocorrência de TMC entre os estudantes procedentes do local do estudo. Esperava-se o contrário, assim como no estudo sobre prevalência de sintomas de ansiedade e depressão realizado com estudantes de Medicina onde o fato de o estudante ser procedente do local do estudo surgiu como fator de proteção para sintomas de depressão $(p<0,05, \mathrm{RP}=0,55, \mathrm{IC}=0,33-0,93)^{(24)}$.

Contudo, com base nos resultados de uma pesquisa com graduandos de enfermagem chineses, em que o bom relacionamento dos estudantes com seus pais e a liberdade para tomarem suas próprias decisões relacionaram-se inversamente com a prevalência de depressão entre os graduandos ${ }^{(15)}$, conjectura-se que a cobrança e a pressão exercidas pelos pais, bem como a menor autonomia dos estudantes que moram com seus familiares, possam justificar o resultado encontrado.

Neste estudo observou-se que quanto melhor a adaptação à universidade, menor a predisposição dos estudantes aos TMC. De forma semelhante, resultados de uma pesquisa com 2.203 estudantes de uma universidade pública do exterior revelaram que os estudantes que vivenciaram experiências negativas na universidade estiveram mais predispostos à piora de sua saúde mental e, quanto mais satisfeitos com os aspectos relacionados à instituição, melhor foi a saúde mental dos universitários ${ }^{(7)}$.

Observaram-se correlações que variaram de fracas a fortes entre os escores de adaptação à universidade e TMC. Destaca-se a dimensão Pessoal que foi a que obteve o menor escore de adaptação e que apresentou correlação forte e negativa com os escores de TMC, evidenciando estreita relação entre os aspectos avaliados por esta dimensão e os sintomas indicativos de TMC.

Em suma, os resultados mostram que o processo de adaptação ao ensino superior merece especial atenção, uma vez que possui repercussões não somente na trajetória acadêmica, mas, também, na saúde dos estudantes. Nesse sentido, ao considerar a complexidade e o dinamismo da temática e a variedade de fatores intervenientes são necessárias pesquisas e ações frente às diferentes demandas estudantis, sejam de saúde, de qualidade de vida e/ou institucionais, a fim de promover um melhor processo de adaptação e, consequentemente, melhoria da saúde, da qualidade de vida, bem como o desenvolvimento das potencialidades dos estudantes ${ }^{(1,25)}$. 


\section{CONCLUSÃO}

Os resultados encontrados nesta pesquisa evidenciaram que os participantes apresentaram um bom nível de adaptação à universidade, especialmente, em relação ao seu envolvimento no curso e projeto vocacional, à satisfação com a instituição que frequentam, às atividades extracurriculares e ao relacionamento interpessoal. Observou-se elevada prevalência de rastreamento positivo para TMC e uma correlação inversa e significativa com adaptação, indicando que quanto melhor a adaptação à universidade, menor a predisposição aos TMC.

Em acréscimo, os resultados apontam a necessidade de intervenções direcionadas à adaptação acadêmica como estratégia de promoção da saúde dos universitários, além de contribuir com o melhor desempenho e o sucesso acadêmicos.

Ressalta-se que este estudo apresenta limitações, tendo em vista a profundidade do assunto e a diversidade de elementos influenciadores. Outra limitação relevante se refere ao desenho transversal que permite estabelecer relações gerais a partir da comparação dos indivíduos no mesmo recorte temporal, no entanto, não possibilita determinar associações do tipo causa-efeito.

Por fim, recomendam-se novas investigações, especialmente, longitudinais para melhor compreensão desta relação e de intervenção que forneçam evidências para o desenvolvimento de ações relacionadas à temática.

\section{REFERÊNCIAS}

1. Oliveira REC, Morais A. Vivências acadêmicas e adaptação de estudantes de uma universidade pública federal do Estado do Paraná. R. Educ. Públ. [Internet]. 2015 [acesso em: 28 nov 2017];24(57),547-68. Disponível em:

http://periodicoscientificos.ufmt.br/ojs/index.php/educacaopublica/article/view/1796.

2. Santos AS, Oliveira CT; Dias ACG. Características das relações dos universitários e seus pares: implicações na adaptação acadêmica. Psicol. teor. prat. [Internet]. 2015 [acesso em: 28 nov 2017];17(1),150-63. Disponível em:

http://pepsic.bvsalud.org/scielo.php?script=sci arttext\&pid=S1516-36872015000100013.

3. Chao RCL. Managing perceived stress among college students: the roles of social support and dysfunctional coping. J Coll Couns [Internet]. 2012 [acesso em: 28 nov 2017];15(1):5-21. Disponível em: http://onlinelibrary.wiley.com/doi/10.1002/j.21611882.2012.00002.x/full.

4. Cestari VRF; Barbosa IV; Florêncio RS; Pessoa VLMP; Moreira TMM. Estresse em estudantes de enfermagem: estudo sobre vulnerabilidades sociodemográficas e acadêmicas. Acta Paul Enferm. [Internet]. 2017 [acesso em: 28 nov 2017]; 30(2):190-6. Disponível em: http://dx.doi.org/10.1590/1982-0194201700029.

5. Santos AAA, Mognon JF, Lima TH, Cunha NB. A relação entre vida acadêmica e a motivação para aprender em universitários. Psicol. esc. educ. [Internet]. 2011 [acesso em: 20 set 2016];15(2):283-90. Disponível em: http://dx.doi.org/10.1590/S141385572011000200010.

6. Ribeiro IM, Patrício ZM, Reis AE, Santos EM. Repercussões do processo ensino-aprendizagem na qualidade de vida-saúde de acadêmicos: entre possibilidades e limitações. Reme, Rev. Min. Enferm. [Internet]. 2010 [acesso em: 20 jun 2016];14(1):96-102. Disponível em: http://www.reme.org.br/artigo/detalhes/93.

7. Byrd DAR, McKinney KJ. Individual, interpersonal, and institutional level factors associated with the mental health of college students. J Am Coll Health [Internet]. 2012 [acesso em: 28 nov 2017];60(3),185-92. Disponível em:

http://www.tandfonline.com/doi/full/10.1080/07448481.2011.584334?scroll=top\&needAccess=true.

8. Benavente SBT, Costa ALS. Respostas fisiológicas e emocionais ao estresse em estudantes de enfermagem: revisão integrativa da literatura científica. Acta Paul Enferm. [Internet]. 2011 [acesso em: 28 nov 2017];24(4):571-6. Disponível em:

http://dx.doi.org/10.1590/S0103-21002011000400019.

9. Marchi KC, Bárbaro AM, Miasso Al, Tirapelli CR. Ansiedade e consumo de ansiolíticos entre estudantes de enfermagem de uma universidade pública. Rev. Eletr. Enf. [Internet]. 2013 [acesso em 15 set 2016];15(3):731-9. Disponível em:

http://dx.doi.org/10.5216/ree.v15i3.18924.

10. Fiorotti KP, Rossoni RR, Borges LH, Miranda AE. Transtornos mentais comuns entre os estudantes do curso de medicina: prevalência e fatores associados. J. bras. psiquiatr. [Internet]. 2010 [acesso em: 20 jun 2016];59(1):17-23. Disponível em: http://www.scielo.br/scielo.php?script=sci arttext\&pid=S0047-20852010000100003\&lng=en. 
11. Silva RS, Costa LA. Prevalência de transtornos mentais comuns entre estudantes universitários da área da saúde. Encontro [Internet]. 2012 [acesso em: 28 nov 2017];15(23):105-12. Disponível em:

http://www.pgsskroton.com.br/seer/index.php/renc/article/view/2473.

12. Vidal CEL, Yañez BFP, Chaves CVS, Yañez CFP, Michalaros IA, Almeida LAS. Transtornos mentais comuns e uso de psicofármacos em mulheres. Cad. Saude Colet. [Internet]. 2013 [acesso em: 28 nov 2017];21(4):457-464. Disponível em:

http://www.scielo.br/scielo.php?script=sci arttext\&pid=S1414-462X2013000400015.

13. Sulkowski ML, Joyce DJ. School psychology goes to college: the emerging role of school psychology in college communities. Psychol Sch [Internet]. 2012 [acesso em: 28 nov 2017];49(8):809-15. Disponível em:

http://onlinelibrary.wiley.com/doi/10.1002/pits.21634/full.

14. Costa EFO, Rocha MMV, Santos ATRA, Melo EV, Martins LAN, Andrade TM. Common mental disorders and associated factors among final-year healthcare students. Rev. Assoc. Med. Bras. [Internet]. 2014 [acesso em: 28 nov 2017];60(6):525-30. Disponível em: http://dx.doi.org/10.1590/1806-9282.60.06.009.

15. Xu Y, Chi X, Chen S, Qi J, Zhang P, Yang Y. Prevalence and correlates of depression among college nursing students in China. Nurse Educ Today [Internet]. 2014 [acesso em: 28 nov 2017];34(6):e7-12. Disponível em:

https://doi.org/10.1016/j.nedt.2013.10.017.

16. Soares AP, Almeida LS, Ferreira JA. Questionário de Vivências Académicas: Versão Integral (Qva) e Versão Reduzida (Qva-R). In Simões MR, et al., coord. Avaliação psicológica: instrumentos validados para a população portuguesa. Coimbra: Quarteto [Internet]. 2006 [acesso em: 10 out 2016];1:101-20. Disponível em: http://hdl.handle.net/1822/12110.

17. Santos KOB, Araujo TM, Pinho PS, Silva ACC. Avaliação de um instrumento de mensuração de morbidade psíquica: estudo de validação do Self-Reporting Questionnaire (SRQ-20). RBSP [Internet]. 2010 [acesso em: 28 nov 2017];34(3):544-60. Disponível em: http://files.bvs.br/upload/S/0100-0233/2010/v34n3/a1881.pdf.

18. Nardelli GG, Gaudenci EM, Garcia BB, Carleto CT, Gontijo LM, Pedrosa LAK. Perfil dos alunos ingressantes dos cursos da área da saúde de uma universidade federal. REAS [Internet]. 2013 [acesso em: 20 mar 2016];2(1):3-12. Disponível em:

http://seer.uftm.edu.br/revistaeletronica/index.php/enfer/article/view/405.

19. Donati L, Alves MJ, Camelo SHH. O perfil do estudante ingressante no curso de graduação em enfermagem de uma faculdade privada. Rev. enferm. UERJ [Internet]. 2010 [acesso em: 28 nov 2017];18(3):446-50. Disponível em:

http://www.facenf.uerj.br/v18n3/v18n3a19.pdf..

20. Silva AO, Cavalcante Neto JL. Associação entre níveis de atividade física e transtorno mental comum entre estudantes universitários. Motricidade [Internet]. 2014 [acesso em: 24 jun 2016];10(1):49-59. Disponível em:

http://dx.doi.org/10.6063/motricidade.10(1).2125.

21. Ansolin AGA, Rocha DLB, Santos RP, Pozzo VCD. Prevalência de transtorno mental comum entre estudantes de psicologia e enfermagem. Arq Cienc Saude [Internet]. 2015 [acesso em: 28 nov 2017]; 22(3):42-5. Disponível em:

http://www.cienciasdasaude.famerp.br/index.php/racs/article/view/83/103.

22. Ferreira CMG; Kluthcovsky ACGC; Cordeiro TMG. Prevalência de Transtornos Mentais Comuns e fatores associados em estudantes de Medicina: um estudo comparativo. Rev. bras. educ. med. [Internet]. 2016 [acesso em: 20 out 2016];40(2):268-77. Disponível em: http://dx.doi.org/10.1590/1981-52712015v40n2e02812014.

23. Ran M-S, Mendez AJ, Leng L-L, Bansil B, Reyes N, Cordero G, Carreon C, Fausto M, Maminta L, Tang M. Predictors of Mental Health Among College Students in Guam: Implications for Counseling. J Couns Dev [Internet]. 2016 [acesso em: 20 out 2016];94:344-55. Disponível em: http://onlinelibrary.wiley.com/doi/10.1002/jcad.12091/full.

24. Vasconcelos TC, Dias BRT, Andrade LR, Melo GF, Barbosa L, Souza E. Prevalência de Sintomas de Ansiedade e Depressão em Estudantes de Medicina. Rev. bras. educ. med. [Internet]. 2015 [acesso em: 15 set 2016];39(1):135-42. Disponível em: http://dx.doi.org/10.1590/1981-52712015v39n1e00042014.

25. Oliveira NRC, Padovani RC. Saúde do estudante universitário: uma questão para reflexão. Ciênc. saúde coletiva [Internet]. 2014 [acesso em: 28 nov 2017];19(3):995-6. Disponível em: http://dx.doi.org/10.1590/1413-81232014193.11042012. 